\title{
Sugar-sensitive neurone responses and sugar feeding preferences influence lifespan and biting behaviours of the Afrotropical malaria mosquito, Anopheles gambiae
}

\author{
Sébastien Kessler • Michèle Vlimant • \\ Patrick M. Guerin
}

Received: 7 July 2014 / Revised: 7 January 2015 / Accepted: 8 January 2015 / Published online: 21 January 2015

(C) Springer-Verlag Berlin Heidelberg 2015

\begin{abstract}
Floral nectar is the main source of carbohydrates for many insects including mosquitoes. Nonetheless, the physiological mechanisms underlying feeding on carbohydrates by the Afrotropical malaria mosquito Anopheles gambiae remain poorly understood. Here, we tested whether sugar sensitivity and sugar feeding preferences correlate with longevity in A. gambiae. We also tested whether feeding females on different sugar diets influences their biting behaviours. Electrophysiological recordings show that sugar neurones on the labella of females are most sensitive to sucrose, mixtures of glucose and fructose, and to melezitose; other sugars tested, including glucose and fructose presented alone, only weakly activate these taste neurones. Mosquitoes survive longest on sucrose, the most preferred sugar. Whereas feeding on a mixture of glucose and fructose is preferred over fructose or glucose alone, fructose supports higher longevity than either glucose or the mixture of the two hexoses. Females that had previously fed on glucose show a stronger biting response than those fed on sucrose, perhaps in an effort to compensate for their lower energetic state. These findings contribute to our
\end{abstract}

Electronic supplementary material The online version of this article (doi:10.1007/s00359-015-0978-7) contains supplementary material, which is available to authorized users.

S. Kessler · M. Vlimant · P. M. Guerin $(\bowtie)$

Institute of Biology, University of Neuchâtel,

Rue Emile-Argand 11, 2000 Neuchâtel, Switzerland

e-mail: patrick.guerin@unine.ch

S. Kessler

e-mail: sebastien.kessler@ncl.ac.uk

Present Address:

S. Kessler

Centre for Behaviour and Evolution, Institute of Neuroscience,

Newcastle University, Newcastle upon Tyne NE1 7RU, UK understanding of the physiological basis of sugar feeding in A. gambiae and indicate how the sugar diet can affect laboratory-reared A. gambiae biting behaviours.

Keywords Sugar taste $\cdot$ Mosquito feeding preferences . Mosquito longevity $\cdot$ Mosquito biting behaviour .

Anopheles gambiae

\section{Introduction}

All animals need a source of macronutrients (i.e. carbohydrates and proteins) to support somatic maintenance and reproduction. Female mosquitoes are unique among animals because their diet is clearly dichotomized into foods which satisfy their requirements for carbohydrate and protein (i.e. blood) consumption. Sugars acquired from nectar are the main source of carbohydrates for female mosquitoes and the only resource consumed by males (Foster 1995). Females of anautogenous mosquito species require sugars in spite of the selective pressure on females living in sugar-scarce habitats to feed only on blood (Gary and Foster 2001).

Females of the malaria mosquito, Anopheles gambiae Giles sensu stricto, with access to plant sugars have a longer lifespan when fed carbohydrates but their biting rate is depressed compared to those with access to blood alone or to plants producing low amounts of nectar (Gary and Foster 2001; Stone et al. 2011, 2012). Sugar is generally preferred over a blood meal by female A. gambiae 2-4 days following emergence, possibly to increase their lifespan (Stone et al. 2011). Most plant nectars found in A. gambiae habitats contain sucrose and/or its hexose hydrolysis products glucose and fructose (Foster 1995; Manda et al. 2007a, b; Nyasembe et al. 2012) and typical sugar concentrations in floral nectar are between 20 and 
$50 \%$ (Foster 1995). Nectar constituents known to influence both mosquito sugar-meal choices and longevity include carbohydrates (Nayar and Sauerman 1971, 1975; Andersson 1992; Ignell et al. 2010; Kessler et al. 2013), amino acids (Ignell et al. 2010; Vrzal et al. 2010) or potentially toxic secondary metabolites such as alkaloids (Ignell et al. 2010; Kessler et al. 2013). However, no studies to date have established whether sugars that are phagostimulatory to $A$. gambiae also result in greater longevity, nor have any studies examined how specific sugar diets might influence mosquito blood feeding behaviours.

Sugars are sensed by insects using taste receptor neurones housed in terminal pore sensilla (Altner and Prillinger 1980). As shown in the winter mosquito, Culiseta inornata, mosquitoes possess sugar-sensitive neurones in sensilla situated on their legs and mouthparts (Pappas and Larsen 1976, 1978). Two types of sensilla have been identified on the external surface of the labella of $C$. inornata: trichoid type 1 (T1) and trichoid type 2 (T2) sensilla (Pappas and Larsen 1976). Each $C$. inornata T1 sensillum houses a neurone responding to mechanical stimulation, a water-sensitive, two salt-sensitive and a sugar-sensitive neurone. T2 C. inornata sensilla are innervated by only three neurones (Pappas and Larsen 1976). The sugar-sensitive neurone in $\mathrm{T} 1$ sensilla triggers the labellar opening response to feed on sucrose (Pappas and Larsen 1976, 1978). In female A. gambiae, labellar T1 sensilla are similar to those of $C$. inornata in that they also house four taste and one mechanoreceptive neurone (Kessler et al. 2013). The sugar-sensitive neurone in A. gambiae $\mathrm{T} 1$ sensilla responds specifically to sucrose and to a mixture of its two hexose constituents, glucose and fructose, at concentrations much lower than those triggering mosquito engorgement on sugars (Kessler et al. 2013). To date, sensitivity of taste neurones to other sugars has not been investigated in A. gambiae and few studies have attempted to correlate the nutritional value of sugars for an animal with the sensory responses of its peripheral taste receptor neurones. It is possible that many sugars can be perceived as such yet not to have a metabolic value; likewise, some sugars that have no taste might still be metabolized (Hassett et al. 1950; Burke and Waddell 2011; Fujita and Tanimura 2011; Stafford et al. 2012).

Here, we used the single sensillum electrophysiological recording technique to characterize the ability of sugarsensitive neurones at the tip of female A. gambiae labella to discriminate between 12 sugars and 1 sugar alcohol. We also evaluated the feeding preferences of $A$. gambiae for the three most important nectar sugars, i.e. sucrose, fructose, glucose and a mixture of the two hexoses, in a two-choice assay. Furthermore, we assessed whether the preferred sugars also resulted in higher longevity of A. gambiae. To test whether sugars found in the diet influence blood feeding, we fed female A. gambiae for 4 or 10 days with sucrose or glucose and measured several behaviours associated with blood feeding including the number of landings, the time spent biting and the average biting time on a heated glass disk.

\section{Materials and methods}

\section{Mosquitoes}

A malaria mosquito, A. gambiae, colony was kept as previously described (Kessler et al. 2013). Larval density was 300 larvae per $400 \mathrm{ml}$ of demineralised water and larvae of all generations were fed with the same amount of Tetramin ${ }^{\circledR}$ fish food in order to standardize mosquito size.

\section{Chemicals}

The trisaccharides 1-kestose, $\mathrm{D}-(+)$-melezitose monohydrate and $\mathrm{D}-(+)$-raffinose, the disaccharides $\mathrm{D}-(+)$-maltose monohydrate, D- $(+)$-sucrose, D- $(+)$-trehalose and D-(+)-turanose, the monosaccharides $\mathrm{D}-(-)$-fructose, $\mathrm{D}-(+)$-glucose monohydrate, L- $(+)$-gulose, methyl- $\alpha$-D-glucopyranoside, methyl$\beta$-D-glucopyranoside and $\mathrm{L}-(-)$-sorbose, and the sugar alcohol D-sorbitol were tested. All were purchased from Sigma-Aldrich (Buchs, Switzerland) at a purity of $\geq 97 \%$. Solutions were kept at $4{ }^{\circ} \mathrm{C}$ for less than 1 week. $\mathrm{KCl}$ was purchased from Merck (Darmstadt, Germany).

Ultrastructure of labellar sensilla in female A. gambiae

Scanning (SEM) and transmission electron microscopy (TEM) procedures were used to characterize trichoid type 1 (T1) and type 2 (T2) sensilla on the labella of female A. gambiae as previously described (Kessler et al. 2013). Ultrathin sections $(1 \mu \mathrm{m})$ of the distal $100 \mu \mathrm{m}$ of the proboscis of three female $A$. gambiae were subjected to TEM to describe the ultrastructure of $\mathrm{T} 2$ sensilla.

Electrophysiological recordings from female A. gambiae labellar sensilla

Electrophysiological recordings from $\mathrm{T} 1$ and $\mathrm{T} 2$ labellar sensilla were made on newly emerged female A. gambiae as previously described (Kessler et al. 2013). A tastePROBE (DTP-02, Syntech, Kirchzarten, Germany) was used in "pass-through" mode, i.e. without compensation for the DC offset, omitting the first 10-20 ms of recordings. The DC offset was reduced by high pass filtering $(100 \mathrm{~Hz})$. Four different experiments were performed.

To search for the presence of water- and sugar-sensitive neurones in $\mathrm{T} 1$ labellar sensilla, nanopure water and $146 \mathrm{mM}$ sucrose (5\%,wt:vol) in $10 \mathrm{mM} \mathrm{KCl}$ were tested on 


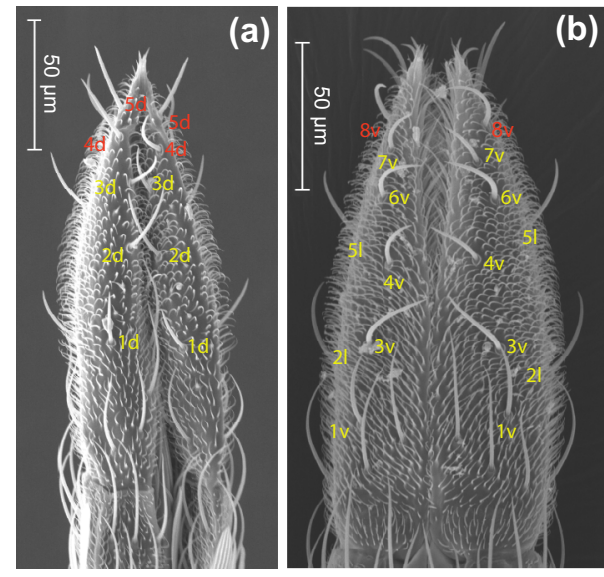

(c)

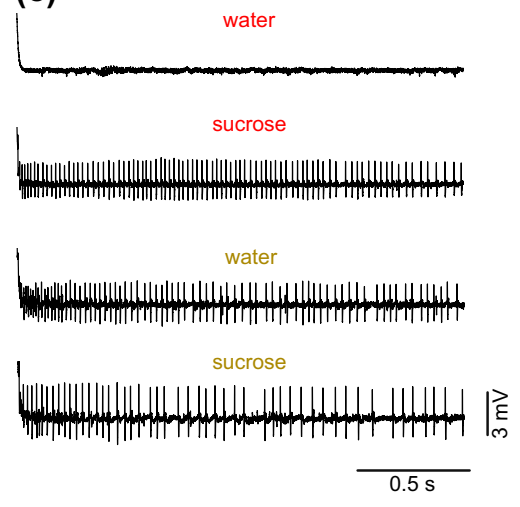

(d)

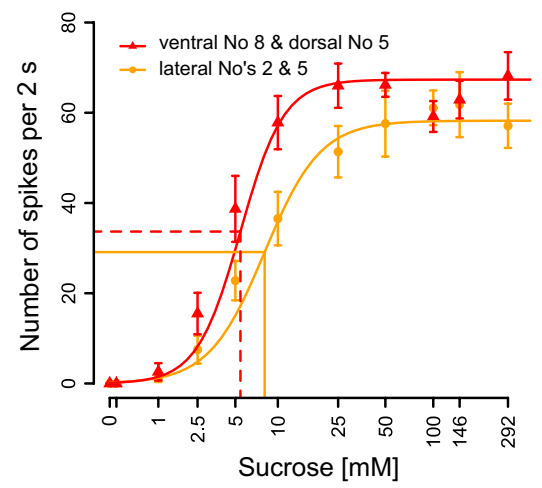

Fig. 1 Scanning electron micrographs of the labella of female $A$. gambiae: a dorsal and b ventral view. Trichoid type 1 (T1) sensilla which house both sugar- and water-sensitive neurones are numbered in yellow and those which house a sugar-sensitive but no water-sensitive neurone are numbered in red; $d$ dorsal, $l$ lateral, $v$ ventral. c Representative electrophysiological responses recorded from the ventral No. 8 and the lateral No. 5 T1 sensilla of A. gambiae to stimulation with water (respectively, the first and third recordings from top) and

$13 \mathrm{~T} 1$ sensilla on each labellum of females. The presence of water- and/or sugar-sensitive neurones within these sensilla was confirmed when recordings from the sensilla of three different mosquitoes showed a response to either of these solutions. Absence of a water-sensitive neurone was confirmed when recordings on at least seven sensilla of seven mosquitoes showed no response to water but responded to sucrose. Water and $100 \mathrm{mM}$ sucrose were also tested on four $\mathrm{T} 2$ sensilla of four different female A. gambiae.

Sucrose at between 0.1 and $292 \mathrm{mM}(10 \%$, wt:vol) in $10 \mathrm{mM} \mathrm{KCl}$ was tested on four T1 sensilla: on the ventral No. 8 and dorsal No. 5 sensilla (i.e. two sensilla which possess a sugar-sensitive neurone but no water-sensitive neurone; Fig. 1a, b) and on lateral Nos. 2 and 5 (i.e. two sensilla which possess both a water- and a sugar-sensitive neurone; Fig. 1b).

The response to $10 \mathrm{mM} \mathrm{KCl}$ of taste neurones within the ventral No. 8 and the dorsal No. 5 sensilla were compared to stimulation with ecologically relevant sugars at $100 \mathrm{mM}$ in $10 \mathrm{mM} \mathrm{KCl}$. The sugars tested were sucrose, fructose, glucose, gulose and maltose which are found in nectars of Kenyan plant species preferred by A. gambiae (Manda et al. 2007a; Nyasembe et al. 2012) and melezitose, trehalose and turanose which are components of homopteran honeydew (Heimpel and Jervis 2005). For comparison, sorbose, a non-nutritive phagostimulant for the black salt marsh mosquito Aedes taeniorhynchus (Nayar and Sauerman 1971), and sorbitol, which is tasteless but nutritionally valuable for Drosophila (Burke and Waddell 2011; Fujita and Tanimura 2011), were also included. to stimulation with $146 \mathrm{mM}$ sucrose in $10 \mathrm{mM} \mathrm{KCl}$ (respectively, the second and fourth recordings from top). d Mean \pm SEM. and fitted mixed effects 3-parameter log-logistic model for the number of spikes generated per $2 \mathrm{~s}$ by sugar-sensitive neurones in ventral No. 8 and dorsal No. 5 (red triangles) and lateral Nos. 2 and 5 T1 sensilla (yellow circles) of female A. gambiae in response to increasing concentrations of sucrose. Continuous and broken straight lines indicate the $\mathrm{ED}_{50}$ values; 6 mosquitoes were tested

To assess the response of the sugar-sensitive neurones within the ventral No. 8 and dorsal No. 5 sensilla to the two hexoses of sucrose, mixtures of glucose and fructose at $5 / 15,15 / 5$ and $10 / 10 \mathrm{mM}$ were compared to 10 and $20 \mathrm{mM}$ glucose or fructose alone and with 5, 10 and $20 \mathrm{mM}$ sucrose. In addition, methyl- $\alpha$-D-glucopyranoside and methyl- $\beta$-D-glucopyranoside mixed with fructose at $10 / 10 \mathrm{mM}$ were tested to gauge the selectivity of the sugar-sensitive neurones. Hexose mixture concentrations were chosen to allow a comparison with $10 \mathrm{mM}$ sucrose, a dose in the maximal sensitivity range of the sugar-sensitive neurones (i.e. near the $\mathrm{ED}_{50}$, see Fig. 1d). In the other experiments, sugars were tested at 100 or $146 \mathrm{mM}$, two concentrations higher than those which evoke the maximal response of the sugar-sensitive neurones (see Fig. 1d).

Spikes were counted in the first $2 \mathrm{~s}$ of recordings made for 4-6 s from T1 sensilla. When increasing concentrations of sucrose were tested, spikes generated in $\mathrm{T} 1$ sensilla by the sugar-sensitive neurone and those generated in response to $10 \mathrm{mM} \mathrm{KCl}$ were differentiated by shape as previously described (Kessler et al. 2013). In all other recordings, the total number of spikes was counted without discriminating between the different units firing. In the case of T2 sensilla, the first $10 \mathrm{~s}$ of recordings of up to $30 \mathrm{~s}$ was analysed due to the strong tonic response pattern of the water-sensitive neurone.

\section{Sugar feeding preferences in A. gambiae}

Five to six-day-old males and females $(20 \pm 3$ of both sexes) fed on $292 \mathrm{mM}$ sucrose, were released into 
Makrolon ${ }^{\circledR}$ cages $(200 \times 260 \times 180 \mathrm{~cm})$ for feeding choice experiments that lasted 21.5 from $3 \mathrm{~h}$ before the end of the scotophase, under colony rearing conditions $\left(28{ }^{\circ} \mathrm{C}, 80 \% \mathrm{RH}, 12: 12 \mathrm{~h}\right.$ light:dark cycle with $2 \mathrm{~h}$ simulated sunset and sunrise). Because $292 \mathrm{mM}$ (10\%, wt:vol) sucrose is a standard solution used to maintain most $A$. gambiae colonies (MR4 2011), sucrose and melezitose were tested at this concentration. The monosaccharides were tested at a concentration two times higher than the sucrose solution (584 mM). A mixture of $292 \mathrm{mM}$ glucose plus $292 \mathrm{mM}$ fructose was also tested and water served as a control. Test solutions were provided in pairs in two 10 -ml vials stoppered by $12 \mathrm{~mm}$ diameter $37-\mathrm{mm}$-long cotton dental rolls (No. 17301 00, Hartmann AG, Neuhausen, Switzerland). The vials were inserted upturned in holes $16 \mathrm{~cm}$ apart on the roof of the cage whereby the dental rolls saturated with the test solutions extended $20 \mathrm{~mm}$ into the cage. Sugar solutions were coloured with blue (E131) or red (E122) food dyes (one drop of $30 \mathrm{mg}$ in $2 \mathrm{ml}$ ). The dyes were interchanged between the test solutions and the position of each solution was alternated in cages between repetitions. Mosquitoes had access to water presented on a saturated cotton pad covering a $45.5 \mathrm{~cm}^{2}$ metal mesh opening on the roof of the cage during choice tests. Mosquitoes were cold anaesthetized after experiments and the solution(s) imbibed by each was determined by visual inspection of the dissected crop and midgut (Ignell et al. 2010) to determine the colour of the abdomen (i.e. red or blue, and purple in the case of mosquitoes that imbibed both solutions). All colour estimations were made by the same person.

The longevity of A. gambiae on different sugar diets

The effect on A. gambiae longevity of feeding on different sugars was tested under the colony rearing conditions. Imagoes of the same generation emerged from dishes containing 200 pupae into Plexiglas ${ }^{\circledR}$ rearing cages $(35 \times 35 \times 55 \mathrm{~cm})$. Cages had an opening $(15 \mathrm{~cm}$ diameter $)$ on one side, closed by nylon netting to provide access and a circular opening ( $12 \mathrm{~cm}$ diameter) on the roof closed by metal netting where mosquitoes had access to a moistened cotton pad. Solutions of either $292 \mathrm{mM}$ sucrose, $584 \mathrm{mM}$ fructose, $584 \mathrm{mM}$ glucose, a mixture of $292 \mathrm{mM}$ glucose plus $292 \mathrm{mM}$ fructose or water were provided on dental rolls in four vials $21 \mathrm{~cm}$ apart on the roof of the cages (as described above under feeding preferences). Sugar solutions and the moistened cotton pad were replaced weekly. The sex ratios among treatments are shown in Table 1. The position of the five cages with the different treatments was changed each day in a circular manner. Dead mosquitoes were counted daily and removed from cages using long sleeved gloves to prevent females from feeding on blood.
Table 1 Sex ratios and total number of mosquitoes exposed to sugar diets in longevity assays

\begin{tabular}{lll}
\hline Sugar diets & Percentage of females/males & $N$ total \\
\hline Fructose & $47 / 53$ & 163 \\
Glucose + fructose & $46 / 54$ & 183 \\
Glucose & $46 / 54$ & 168 \\
Sucrose & $43 / 57$ & 165 \\
Water & $57 / 43$ & 192 \\
\hline
\end{tabular}

Body mass and biting behaviour as a function of sugar diet

The biting behaviour of female A. gambiae that had fed previously on glucose or sucrose was tested 4 and 10 days post emergence by quantifying the number of landings and time spent biting on a heated glass disk (see below). Biting is defined here as the act of attempting to pierce the substrate with its mouthparts. The dry mass of these females, as an indicator of their energetic state, and their body water content was also recorded after testing their biting response.

For this behavioural assay, imagoes emerged from dishes containing 200 pupae into Plexiglas ${ }^{\circledR}$ rearing cages $(200 \times 260 \times 180 \mathrm{~mm}$ high $)$. In the days before testing their biting response, imagoes had access ad libitum to water and to either a $292 \mathrm{mM}$ sucrose (10\%, wt:vol) or a $584 \mathrm{mM}$ glucose solution on dental rolls in two vials on the roof of the cage (as described above under feeding preferences). The day before testing, females were individually deprived of water and sugar for $12 \mathrm{~h}$ in glass vials at $95 \% \mathrm{RH}$ under colony temperature and light conditions after which they were allowed to feed for $30 \mathrm{~min}$ on a filter paper (No. 10311 807, Whatman Schleicher and Shuell, Dassel, Germany) treated with $600 \mu \mathrm{l}$ of a $292 \mathrm{mM}$ sucrose or a $584 \mathrm{mM}$ glucose solution (i.e. on the same diet they had been fed). Sugar-engorged females were selected to measure their biting behaviour 3-6 $\mathrm{h}$ after feeding on the filter paper during which time they were held individually in the same glass vials at $95 \% \mathrm{RH}, 25^{\circ} \mathrm{C}$ and 14 lux.

The biting behaviour of females was measured using a warm body assay (Kröber et al. 2010). The responses of individuals was measured for $5 \mathrm{~min}$ to a sandblasted glass disk heated to $35 \pm 0.5{ }^{\circ} \mathrm{C}$ inserted into a Makrolon ${ }^{\circledR}$ cage $(200 \times 260 \times 180 \mathrm{~mm}$ high $)$ holding a female. The biting behaviour was measured in a climate chamber at $25^{\circ} \mathrm{C}$, $80 \% \mathrm{RH}$ and 14 lux, $5 \mathrm{~h}$ into the scotophase. A female was released into test cages at least $10 \mathrm{~min}$ before the start of an experiment. A 0.2-s pulse of pure $\mathrm{CO}_{2}$ (volume $6.25 \mathrm{ml}$ ) from a pressurized cylinder (Carbagas, Gümligen, Switzerland) was released inside the cage using a solenoid valve to activate the mosquito just before the heated glass disk was introduced. The mosquito was filmed at 60.1 image frames/s with a GigE Monochrome Camera (DMK 
23G618, The Imaging Source, Bremen, Germany) mounted on a rail behind the cages so that the $60 \mathrm{~mm}$ diameter glass disk represented 460 pixels. A diode array (CVI STAR-RL100/69-IR850-12V, Stemmer Imaging AG, Pfäffikon, Switzerland) provided infrared light $(850 \mathrm{~nm})$. A foot switch synchronized $\mathrm{CO}_{2}$ release and switching on the camera.

Females which landed on the heated glass disk were detected using the "analyze particles" tool of ImageJ (Rasband 2011). Each picture was transformed into an 8 bit grayscale representation from which a black and white image was generated by adjusting the image threshold to $0 / 76$. Each particle of between 120 and $500 \mathrm{px}^{2}$ was counted as a female. The number of landings and the total time spent biting by mosquitoes were quantified using Matlab (Matlab 8.0, The MathWorks Inc., Natick, MA, 2012). A fresh landing was counted each time more than 10 consecutive frames (166 ms) without a mosquito separated frames with a mosquito. A landing was counted when a mosquito was detected for more than 10 consecutive frames on the heated glass disk. Female A. gambiae only land on the heated glass disk to bite and were never observed resting on it. The time spent biting was quantified by multiplying the number of frames on which a mosquito was detected by the frame length $(16.6 \mathrm{~ms})$. The average biting time was calculated as the total time spent biting divided by the number of landings. Females were anaesthetized on ice immediately after the biting test and their wet mass was recorded using a M3 Microbalance (reading precision of $\pm 1 \mu \mathrm{g}, 1 \mathrm{SD}=2 \mu \mathrm{g}$; Mettler, Greifensee, Switzerland). The dry mass was recorded after desiccation overnight at $70{ }^{\circ} \mathrm{C}$ in an oven. Body water content corresponded to the difference between the wet mass measured immediately after the biting experiment and the dry mass.

\section{Statistical analysis of data}

All statistical analyses and graphical representations were performed with R 3.1.1 (R Development Core Team 2014). The number of spikes as a function of increasing concentrations of sucrose was assessed using a 3-parameter loglogistic mixed model that includes the sensilla as a fixed factor and the mosquitoes as a random variable. We tested for differences between sensilla by pooling them into one or two factor levels. We then fitted the simplified models to the data and tested if the change in deviance between the two models was significant (Crawley 2007). Models were fitted with the nlsList command in the nlme package (Pinheiro et al. 2014).

The number of spikes generated to $100 \mathrm{mM}$ of each sugar tested was compared to the number generated in response to $10 \mathrm{mM} \mathrm{KCl}$ using a generalized linear mixed model (GLMM) with a negative binomial error distribution and a log link function fitted with the glmadmb command in the glmmADMB package (Skaug et al. 2014). The number of spikes was treated as the dependent variable, whereas the sugars and their concentrations in $\mathrm{KCl}$ were used as fixed factors with mosquitoes as a random variable. A similar GLMM was also fitted to the number of spikes recorded from taste neurones in response to $\mathrm{KCl}$, glucose, fructose, $\alpha$ and $\beta$-methyl-glucosides, mixtures of the monosaccharides and sucrose with the sugar solutions as a fixed factor and the mosquitoes as a random variable.

A choice index (CI) according to Ignell et al. (2010) was used for graphical representation of the sugar feeding preferences. The number of mosquitoes that chose either sugar diet A or B was analysed by a generalized linear model (GLM) with a quasi-binomial error distribution and a logit link function. The number of mosquitoes that chose a particular diet was treated as the dependent variable with sugar diets as the fixed factor. GLMs with binomial error distributions were fitted to the number of unfed mosquitoes as well as to the number of mosquitoes that fed on both solutions. Mosquito longevity as a function of sugar diet was analysed with a Cox proportional-hazards model in the survival R package (Therneau 2014).

Differences between dry mass and body water content of mosquitoes were subjected to 2-way ANOVAs with sugar diet and age as the explanatory factors. Separate 2-way ANOVAs were employed to test the effects of sugar diet and age on the average biting time and the time spent biting by mosquitoes on the heated glass disk. The effects of sugar diet and age on the number of landings were analysed by a GLM with a quasi-Poisson error distribution and a log link function. All models were checked for the appropriate distribution of residuals. A $95 \%$ confidence interval level was set for all analyses.

\section{Results}

The sensitivity of labellar taste neurones to sugars in female A. gambiae

The first set of experiments comprehensively define the sugar sensitivities of taste neurones in sensilla on the labella of female A. gambiae. The subset of sugars selected for this study were tested on ventral 8 and dorsal $5 \mathrm{~T} 1$ labellar sensilla as they do not possess a water-sensitive neurone, contrary to the $\mathrm{T} 1$ sensilla situated more proximally on the labellum (Fig. 1a-c). The labellar sugar neurones of female A. gambiae were most sensitive to changes in low concentrations of sucrose: the $\mathrm{ED}_{50}$ for the spike frequency generated by the sugar neurones of the ventral 8 and dorsal $5 \mathrm{~T} 1$ sensilla is significantly lower $(6.45 \mathrm{mM})$ than for the sugar neurones of the lateral sensilla Nos. 2 and 5 (9.12 mM, $P<0.001$; Fig. 1d). In the ventral 8 and dorsal 
(a)

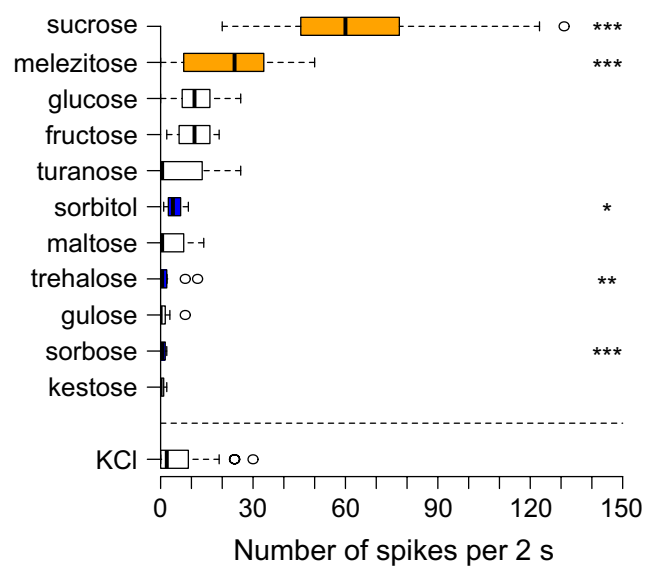

(b)

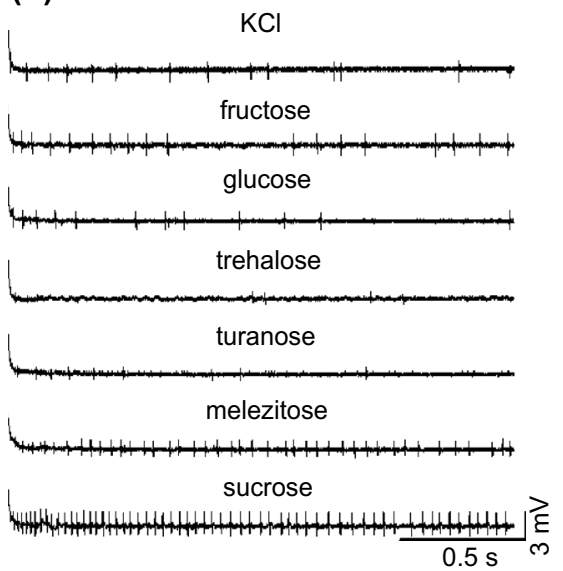<smiles>CCC1CC2C(C)C(C)CC(CC)C2C(CC)C1CC</smiles><smiles>CCC1CC2CC(CC)C3C(C)C(C)C(CC)C3CC2C(CC)C1C</smiles>

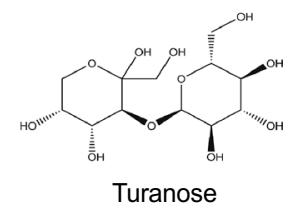

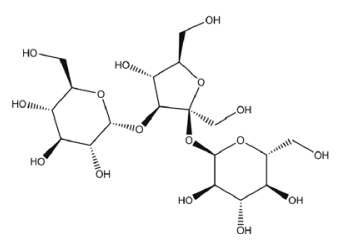

Melezitose

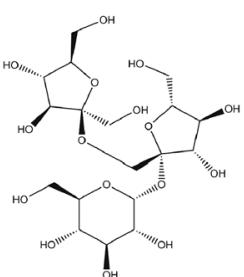

Kestose
Fig. 2 Box plots of the mean number of spikes per $2 \mathrm{~s}$ generated by sugar-sensitive neurones housed in ventral No. 8 and dorsal No. 5 trichoid type 1 (T1) labellar sensilla of female A. gambiae (see Fig. 1) in response to stimulation with $10 \mathrm{mM} \mathrm{KCl}$ and, in increasing order of activity, to $100 \mathrm{mM}$ kestose, sorbose, gulose, trehalose, maltose, sorbitol, turanose, fructose, glucose, melezitose and sucrose diluted in $10 \mathrm{mM} \mathrm{KCl} \mathrm{(a).} \mathrm{Each} \mathrm{sugar} \mathrm{was} \mathrm{tested} \mathrm{on} \mathrm{separate} \mathrm{mosquitoes.} \mathrm{Only}$ those taste neurones responding to $100 \mathrm{mM}$ sucrose in $10 \mathrm{mM} \mathrm{KCl}$, tested as a positive control at the end of the stimulation series, were included in the analysis. $\mathrm{KCl}$ and sucrose control values are from the pooled data on all mosquitoes tested. Melezitose and sucrose were

$5 \mathrm{~T} 1$ sensilla, only melezitose and sucrose at $100 \mathrm{mM}$ in $10 \mathrm{mM} \mathrm{KCl}$ elicited spike frequencies significantly higher than the response to $\mathrm{KCl}$ alone (negative binomial GLMM, $P<0.001$ in both cases; Fig. 2).

Spike frequencies recorded in response to 10 and $20 \mathrm{mM}$ glucose, fructose, methyl- $\beta$-D-glucopyranoside and methyl$\alpha$-D-glucopyranoside hardly activated the sugar-sensitive neurones (responses were not significantly higher than to $10 \mathrm{mM} \mathrm{KCl}$ alone; Fig. 3). Synergism occurred between glucose and fructose as the 10/10,5/15 and 15/5 mixtures of glucose and fructose activated the sugar-sensitive neurones above the response to $\mathrm{KCl}$ alone (negative binomial GLMM, $P$ values $<0.001$ ), but at frequencies lower than the only sugars to evoke a spike frequency significantly higher than the response to $10 \mathrm{mM} \mathrm{KCl}$ (in orange). $100 \mathrm{mM}$ sorbose, trehalose and sorbitol inhibited the response to $10 \mathrm{mM} \mathrm{KCl}$ (in blue; negative binomial GLMM, $* P<0.05, * * P<0.01, * * * P<0.001)$. Between 1 and 2 sensilla from 3-13 mosquitoes were tested per treatment. Box plots represent the median (black bars), the lowest and the highest data points still within 1.5 of the interquartile range (whiskers) and the outliers (circles). b Representative 2-s electrophysiological responses recorded from neurones in ventral No. 8 and dorsal No. 5 $\mathrm{T} 1$ sensilla to $100 \mathrm{mM}$ sugar solutions in $10 \mathrm{mM} \mathrm{KCl}$. Structures of some of the sugars tested are depicted at the bottom

in response to 10 or $20 \mathrm{mM}$ sucrose $(P$ values $<0.05)$. In addition, the equimolar $10 / 10 \mathrm{mM}$ mixture of glucose and fructose generated a significantly higher response than the $15 / 5$ mixture $(P<0.01)$ and a higher but not significantly different spike frequency than to the $5 / 15 \mathrm{mM}$ mixture $(P=0.168>0.05)$.

This interaction also occurred with the mixture of methyl- $\alpha$-D-glucopyranoside and fructose (Fig. 3) as the equimolar mixture of $10 \mathrm{mM}$ fructose and methyl- $\alpha-\mathrm{D}-$ glucopyranoside activated the sugar-sensitive neurones above the responses to $\mathrm{KCl}(P<0.001)$. However, this response was lower than that for the $10 / 10 \mathrm{mM}$ mixture of glucose and fructose $(P<0.05)$ and lower than for the 

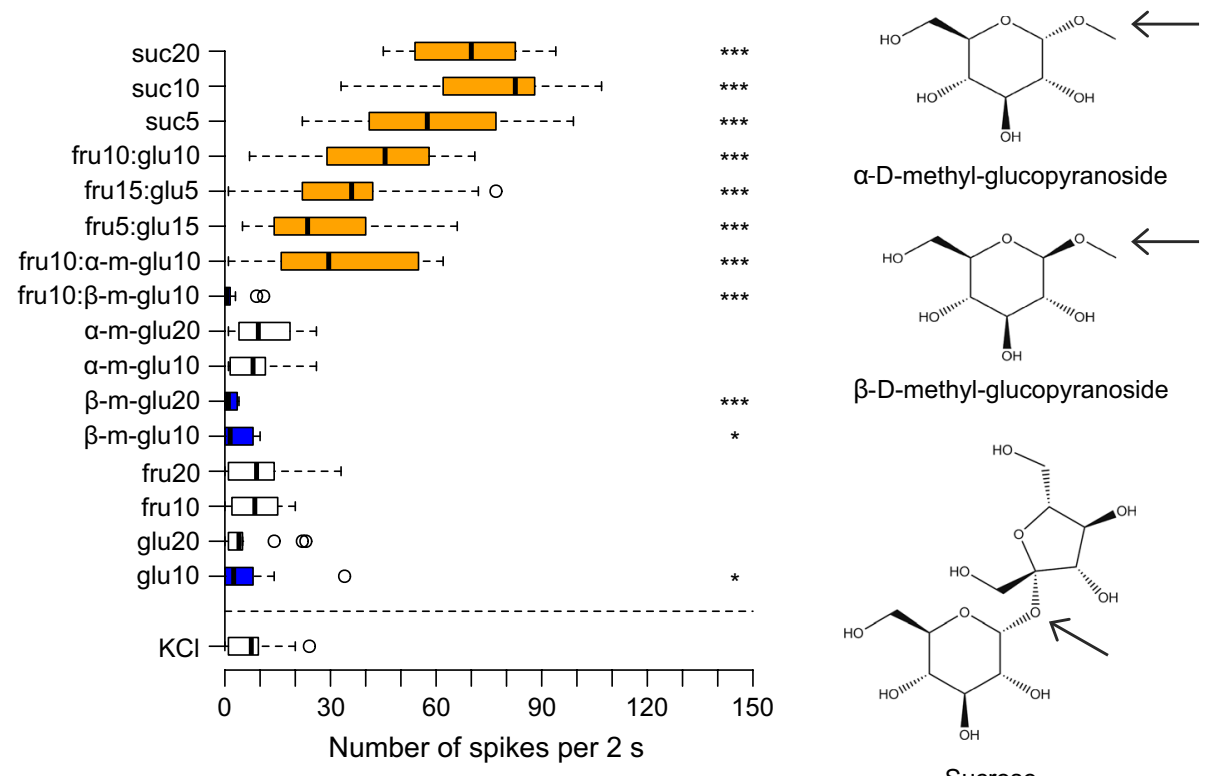

Sucrose

Fig. 3 Box plots of the mean number of spikes generated per $2 \mathrm{~s}$ by sugar-sensitive neurones in ventral No. 8 and dorsal No. 5 trichoid type 1 (T1) labellar sensilla of female A. gambiae (see Fig. 1) in response to stimulation with $10 \mathrm{mM} \mathrm{KCl}, 10$ (glu10) and $20 \mathrm{mM}$ glucose (glu20), 10 (fru10) and $20 \mathrm{mM}$ fructose (fru20), 10 ( $\beta-\mathrm{m}$ glu10) and $20 \mathrm{mM}$ methyl- $\beta$-D-glucopyranoside ( $\beta$-m-glu20), 10 $(\alpha-\mathrm{m}$-glu10) and $20 \mathrm{mM}$ methyl- $\alpha$-D-glucopyranoside $(\alpha-\mathrm{m}$-glu20), $10 \mathrm{mM}$ fructose with $10 \mathrm{mM}$ methyl- $\beta$-D-glucopyranoside (fru $10 / \beta$ $\mathrm{m}$-glu10), $10 \mathrm{mM}$ fructose with $10 \mathrm{mM}$ methyl- $\alpha$-D-glucopyranoside (fru10/ $\alpha$-m-glu10), $5 \mathrm{mM}$ fructose with $15 \mathrm{mM}$ glucose (fru5/glu15),

three concentrations of sucrose tested $(P$ values $<0.001)$. By contrast, the spike frequency recorded in response to equimolar mixtures of $10 \mathrm{mM}$ fructose and methyl- $\beta$ D-glucopyranoside was significantly lower than for $\mathrm{KCl}$ $(P<0.001)$.

Contrary to T1 sensilla, the ultrastructure studies show that $\mathrm{T} 2$ sensilla are not typical gustatory sensilla with a terminal pore as described in Altner and Prillinger (1980) (Fig. 4a-e). T2 sensilla are $5 \mu \mathrm{m}$ long arising from a cylindrical socket and are found proximally on the ventral and dorsal sides of each labellum at a distance of about $40 \mu \mathrm{m}$ from the tip of the labella (Fig. 4a, b). Two neurones innervate these sensilla: one terminates in a tubular body just below the shaft and a second extends its single dendrite to the tip of the hair (Fig. 4c-e). A prominent groove is present on the cuticular surface (Fig. 4b, e). T2 sensilla house a neurone responding to mechanical stimulation and another responding to water which is not affected by $100 \mathrm{mM}$ sucrose (Fig. 4f, g). These findings show that, in female A. gambiae, neurones in labellar T1 sensilla but not in $\mathrm{T} 2$ sensilla are tuned to sense changes in low concentrations of sucrose and to mixtures of its two hexose constituents, glucose and fructose, all constituents of nectar.
$15 \mathrm{mM}$ fructose with $5 \mathrm{mM}$ glucose (fru15/glu5), $10 \mathrm{mM}$ fructose with $10 \mathrm{mM}$ glucose (fru10/glu10), and to 5,10 or $20 \mathrm{mM}$ sucrose, each diluted in $10 \mathrm{mM} \mathrm{KCl}$. Responses significantly higher than the response to $10 \mathrm{mM} \mathrm{KCl}$ are depicted in orange and those significantly lower are in blue (negative binomial GLMM, $* P<0.05$, *** $P<0.001$ ). Two sensilla from 4 to 10 mosquitoes were tested per treatment. For explanation of box plots, see legend to Fig. 2. Arrows on the molecular structures on the right indicate the $\alpha$ and $\beta$ positions of the anomeric groups of the methyl-glucopyranosides

\section{Sugar feeding preferences in A. gambiae}

We found that A. gambiae females exhibited specific preferences for sugar diets in the following order: sucrose $>$ glucose plus fructose $>$ fructose $>$ glucose $>$ water (quasibinomial GLM, $\mathrm{rd}=50.208, d f=40, P$ values $<0.01$; Fig. 5). When offered a choice between sucrose and a glucose plus fructose mixture, $67.5 \%$ of female A. gambiae who made a choice preferred to feed on sucrose whereas $32.5 \%$ chose the mixture. This difference was significant $(P<0.01)$. Female A. gambiae also preferred to feed on sucrose rather than on melezitose at the same concentration $(P<0.001)$. This preference was comparable to what was observed when sucrose was compared to a twice more concentrated solution of fructose $(P=0.674)$ or glucose $(P=0.627$; Fig. 5).

The percentage of females that fed on both solutions was significantly higher when presented a choice between sucrose and a mixture of glucose and fructose $(15.93 \%)$ than in the other choices tested (between 0 and $1.16 \%$; binomial GLM with, $\mathrm{rd}=13.544, d f=40, P<0.05$; Fig. 5). Overall, fewer female A. gambiae choose to feed when given a choice between glucose and water $(51.25 \%)$ 

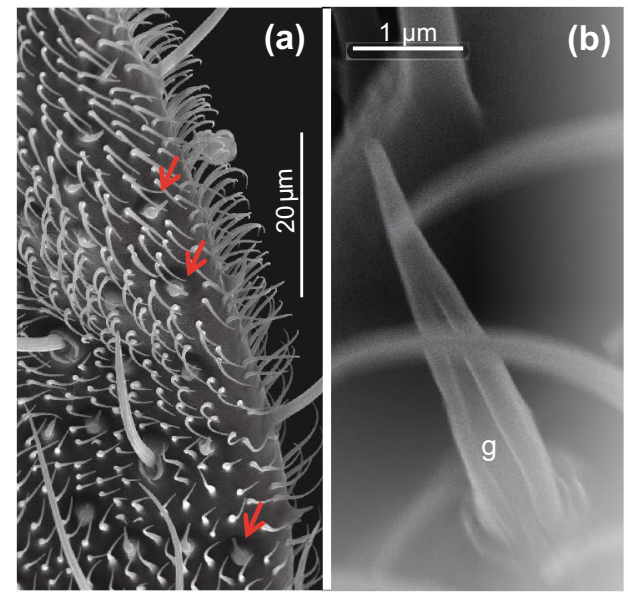

(f)

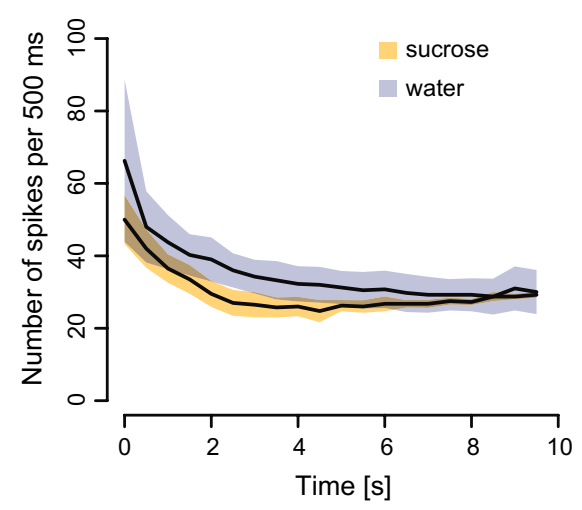

Fig. 4 Scanning electron micrograph of the external surface of a labellum with arrows indicating trichoid type 2 (T2) sensilla visible between the microtrichia and the much bigger trichoid type 1 (T1) sensilla (a). Scanning electron micrograph of a T2 sensillum (b) and transmission electron micrographs of transverse sections at the base (c), above the ciliary root (d) and half way up the sensillum (e); $c$ cuticle, $d$ dendrite, $e v$ enveloping cells, $g$ groove, $s h$ sheath, $t b$ tubular body. Adaptation curves (mean \pm s.e.m., $N=4$ ) showing action potential frequency recorded from $\mathrm{T} 2$ sensilla of A. gambiae at

than in tests with other treatments (between 97.53 and $77.22 \%$; binomial GLM, rd 45.359, $d f 40, P$ values $<0.01$ ). The sugar-meal preferences of male $A$. gambiae were similar to those of females (Supplementary material, Fig. S1).

The longevity of A. gambiae on different sugar diets

We then assessed how sugar solutions support A. gambiae longevity. Sugar diets contributing to A. gambiae female longevity were in decreasing order, $292 \mathrm{mM}$ sucrose $=584 \mathrm{mM}$ fructose $>292 \mathrm{mM}$ glucose plus $292 \mathrm{mM}$ fructose $>584 \mathrm{mM}$ glucose $>$ water (Cox proportional-hazards regression model, $\chi^{2}=499.1, d f=4$, $P<0.001$; Fig. 6). Water did not allow females to survive more than 4 days, shorter than for any of the sugars
$500 \mathrm{~ms}$ intervals over 10-s stimulation with water or $100 \mathrm{mM}$ sucrose (f). Representative 1-s electrophysiological responses to stimulation with water and $100 \mathrm{mM}$ sucrose (g respectively, first and second recordings from top) and during stimulation with water accompanied by mechanical stimulation (mech.) where the sensillum was deflected with the electrode filled with water (g third and fourth recordings from top): the water-sensitive neurone is silenced when the mechanoreceptor neurone is activated but fires again when the sensillum is restored to its initial position

$(P<0.001$ in all cases). Female longevity on sugar treatments differed significantly $(P<0.01)$, except between fructose and sucrose $(P=0.64)$. Maximum female longevity of 28 days was observed on sucrose and fructose. The longevity of male A. gambiae was affected in the same manner by sugar diets as females (Supplementary material, Fig. S2).

Female A. gambiae body mass as a function of sugar diet

To provide an indicator of their energetic state, the dry mass and the body water content of females with access to the preferred sucrose diet or the less preferred glucose diet were quantified after the biting test (below). Access to sucrose was significantly better in allowing 


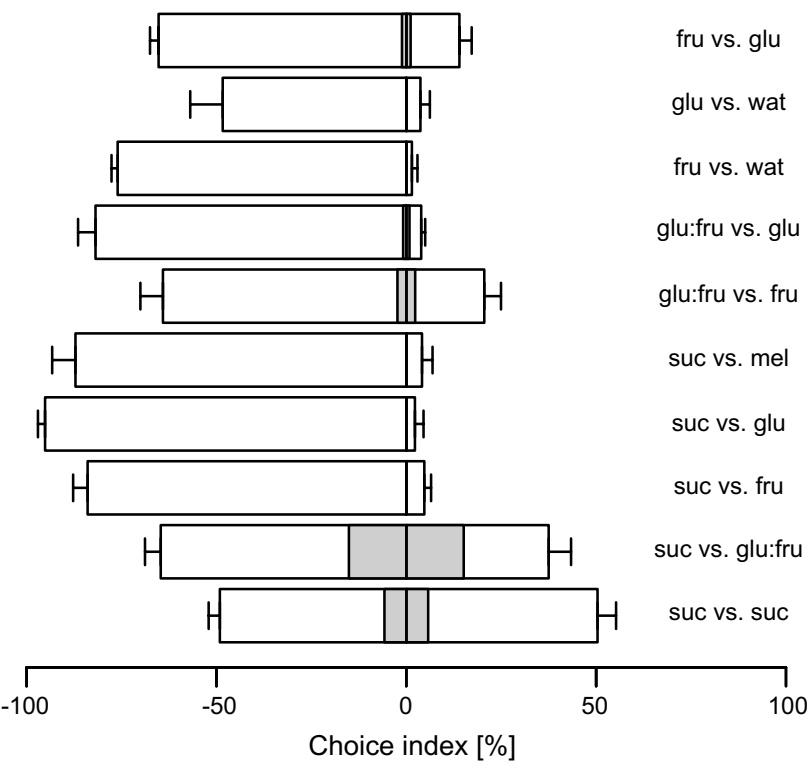

Fig. 5 Mean \pm s.e.m. of feeding preferences by female A. gambiae offered sugar solutions in paired choice tests: $584 \mathrm{mM}$ fructose versus $584 \mathrm{mM}$ glucose; $584 \mathrm{mM}$ glucose or $584 \mathrm{mM}$ fructose versus water; $292 \mathrm{mM}$ glucose with $292 \mathrm{mM}$ fructose versus $584 \mathrm{mM}$ glucose or $584 \mathrm{mM}$ fructose; $292 \mathrm{mM}$ sucrose versus $292 \mathrm{mM}$ melezitose or $584 \mathrm{mM}$ glucose or $584 \mathrm{mM}$ fructose; $292 \mathrm{mM}$ sucrose versus $292 \mathrm{mM}$ glucose with $292 \mathrm{mM}$ fructose or the same solution of $292 \mathrm{mM}$ sucrose. Each choice test consisted of 4-7 replicates with $20 \pm 3$ females in each. Percentages of mosquitoes tested that fed on both solutions are in grey

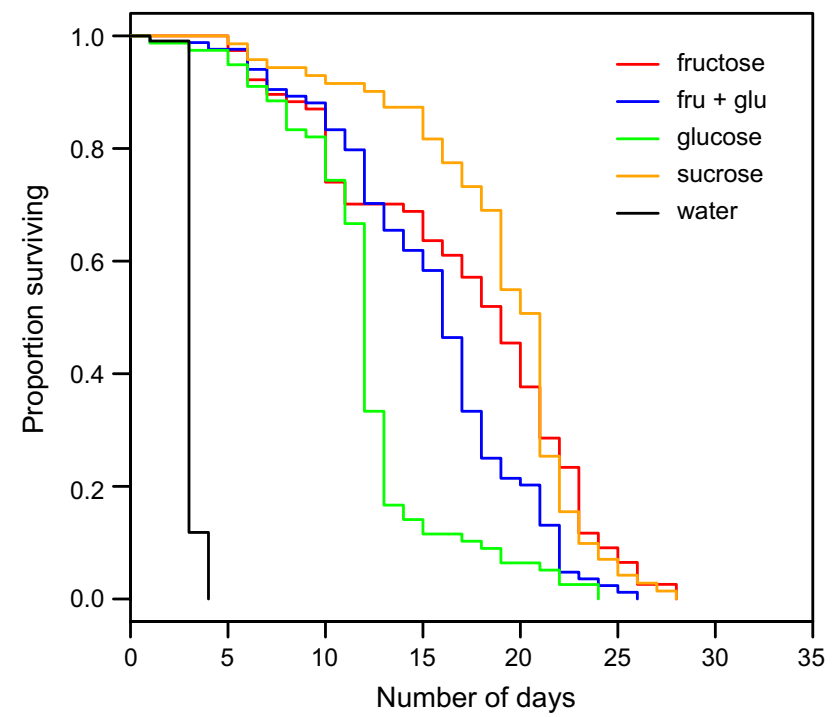

Fig. 6 Survival curves in days for female A. gambiae (between 71 and 110 mosquitoes tested per treatment) fed on $584 \mathrm{mM}$ fructose, $584 \mathrm{mM}$ glucose, $292 \mathrm{mM}$ glucose with $292 \mathrm{mM}$ fructose, $292 \mathrm{mM}$ sucrose or water

female A. gambiae to accumulate energy reserves than access to glucose. Females fed on sucrose had a higher dry mass $(0.45 \pm 0.01 \mathrm{mg}$; mean \pm s.e.m. $)$ than those (a)

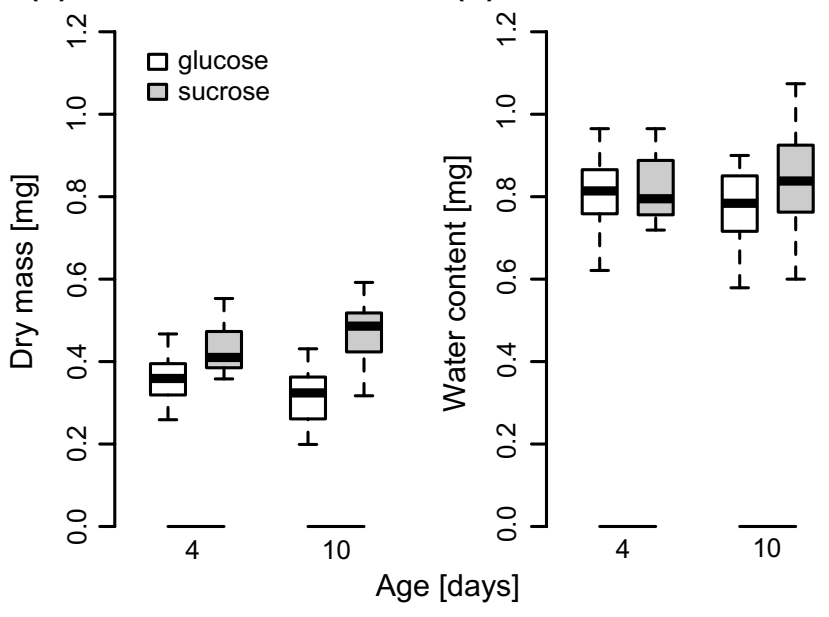

Fig. 7 Dry mass (a) and body water content (b) of 4 and 10-day-old female A. gambiae fed on glucose (white) and on sucrose (grey) following biting tests (see Fig. 8). Data are from 43 and 41 mosquitoes for the glucose and the sucrose diets, respectively. For explanation of box plots, see legend to Fig. 2

fed on glucose $(0.34 \pm 0.01 \mathrm{mg})$ with a significant interaction between sugar diet and age (2-way ANOVA, effect of sugars $\mathrm{F}_{1,80}=58.4, P<0.001$; effect of age $\mathrm{F}_{1,80}=0.135, P=0.71$; effect of the interaction $\mathrm{F}_{1,80}=10.4, P<0.01$; Fig. 7a). The dry mass was lower for 10-day-old females $(0.31 \pm 0.01 \mathrm{mg})$ than for 4-day-old females $(0.36 \pm 0.1 \mathrm{mg})$ fed on glucose but higher for 10-day-old $(0.47 \pm 0.02 \mathrm{mg})$ than for 4-day-old $(0.43 \pm 0.01 \mathrm{mg})$ females fed on sucrose. Females fed on sucrose also had a higher body water content $(0.83 \pm 0.02 \mathrm{mg})$ than those fed on glucose $\left(0.79 \pm 0.01 \mathrm{mg}\right.$; 1-way ANOVA, $\mathrm{F}_{1,82}=4.066$, $P=0.047$; Fig. $7 \mathrm{~b}$ ), but body water content was not dependent on age and no significant interaction was found between sugar diet and age.

Prior to the biting test (below), females were fed on a $292 \mathrm{mM}$ sucrose or a $584 \mathrm{mM}$ glucose solution (i.e. on the same diet they had previously fed on). Whereas some 78 and $84 \%$ of 4-day-old females engorged, respectively, on sucrose and glucose, $91 \%$ of females fed on sucrose but only $34 \%$ on glucose at 10 days old.

Biting responses of female A. gambiae as a function of sugar diet

The biting behaviours of female A. gambiae fed on sucrose and on the less suitable glucose diet were compared. Females were less likely to land (quasi-Poisson GLM, rd $=879.9, d f=108, P<0.05)$ and spent less time biting (1-way ANOVA, $\mathrm{F}_{1,108}=7.024, P<0.01$ ) when fed with sucrose than with glucose (Fig. $8 \mathrm{a}, \mathrm{b}$ ). The average 

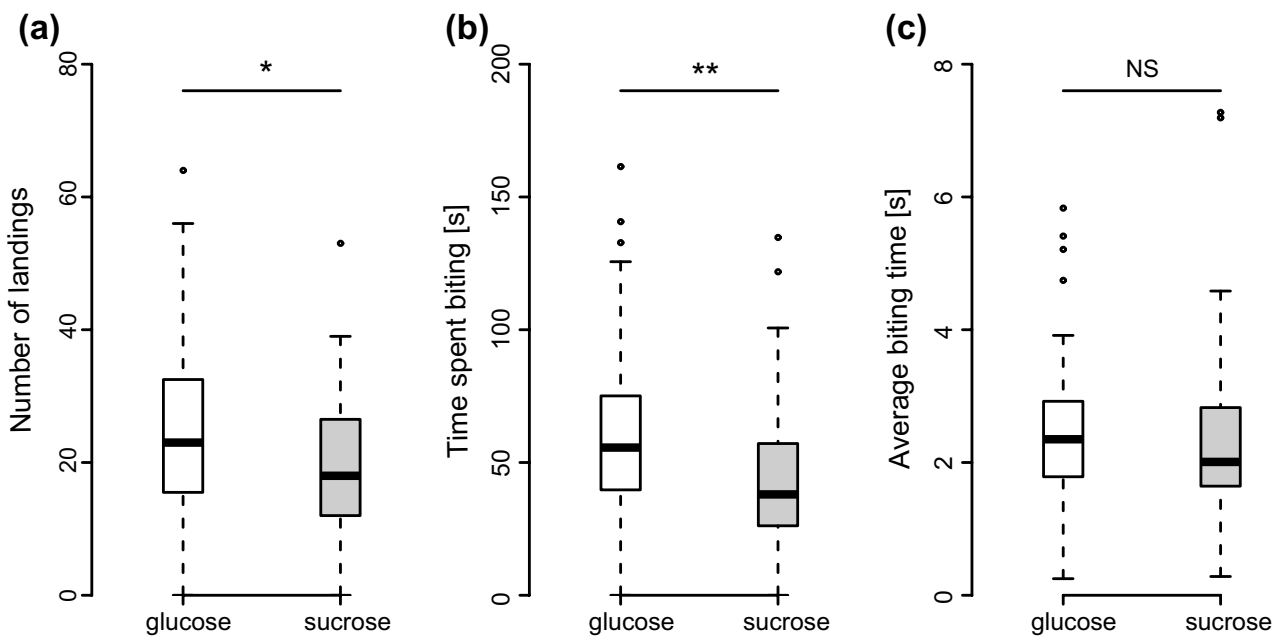

Fig. 8 Number of landings (a quasi-Poisson GLM, $* P<0.05$ ), time spent biting (b 1 -way ANOVA, $* * P<0.01$ ) and average biting time (c 1-way ANOVA, $N S$ non-significant) over $5 \mathrm{~min}$ by female $\mathrm{A}$. gambiae (pooled data of 4- and 10-day-old mosquitoes) on a glass disk heated to skin temperature. Prior to tests, the mosquitoes were fed on $292 \mathrm{mM}$ sucrose or $584 \mathrm{mM}$ glucose. Data are from 55 mosquitoes for both sugar diets. For explanation of box plots, see legend to Fig. 2 biting time was not affected by sugar diet (1-way ANOVA, $\mathrm{F}_{1,103}=0.114, P=0.736>0.05$; Fig. $8 \mathrm{c}$ ). The effect of age and the interaction between sugar diet and age were not significant and were removed from these three statistical models as changes in deviance between the maximal and minimal adequate statistical models were not significant (Crawley 2007).

\section{Discussion}

Neurophysiology of sugar-sensitive neurones in $A$. gambiae

Sucrose, mixtures of fructose and glucose and, to a lesser extent, melezitose are the strongest sugar-sensitive neurone activators from the subset of sugar solutions tested on the T1 labellar tip sensilla of the African malaria mosquito. By comparison, glucose and fructose alone and the other sugars tested represent weak stimuli. Our data indicate that labellar sugar-sensitive neurones are tuned to detect constituents of floral nectar-sucrose and mixtures of fructose and glucose-of plant species growing in the habitat of A. gambiae (Manda et al. 2007a, b; Nyasembe et al. 2012). Although melezitose is scarce in floral and extrafloral nectars, it is a constituent of homopteran honeydews (Heimpel and Jervis 2005) and provides an alternative potential source of energy for A. gambiae (Gary and Foster 2004).

Mixtures of D-fructose and D-glucose, the two hexoses of sucrose, activate the sugar-sensitive neurones in female A. gambiae. This 'synergism' might reflect a mechanism common in other nectar feeding insects. For example, increased responsiveness of mouthpart sugarsensitive neurones to mixtures of glucose and fructose also occurs in the honeybee (Whitehead and Larsen 1976) and in the blowfly Phormia regina (Omand and Dethier 1969). Since methyl- $\alpha$-D-glucopyranoside with fructose activates the sugar-sensitive neurones of $A$. gambiae and methyl- $\beta$-D-glucopyranoside mixed with fructose does not, this could reflect a particular ability of the mosquito to sense the $\alpha$ anomeric form of the glucopyranose which is a constituent of the sucrose molecule (Fig. 3).

Eight putative sugar receptors (Srs) have been identified in A. gambiae by sequence homology with Drosophila melanogaster (Kent and Robertson 2009). The response pattern to the subset of sugars tested here on two labellar taste neurones of female A. gambiae differs to what is known in male Drosophila. In the fruit fly, $100 \mathrm{mM}$ maltose and methyl- $\alpha$-D-glucopyranoside elicit strong responses from sugar-sensitive neurones in L-type labellar sensilla, whereas turanose and trehalose elicit only moderate responses (Dahanukar et al. 2007). No direct orthology has been established between the Srs of Drosophila and A. gambiae (Kent and Robertson 2009), a factor that could explain differences in sweet taste sensitivity between the two species. Nonetheless, as in Anopheles, sucrose appears to be the best activator of labellar sugar-sensitive neurones of Drosophila and monosaccharides do not evoke strong responses (Dahanukar et al. 2007). The sensitivity of sugar taste neurones in male A. gambiae remains to be characterized. 
The relation between feeding preferences and sensitivity of taste receptor neurones in A. gambiae

As predicted by the electrophysiological recordings, sucrose and the mixture of its hexose constituents glucose and fructose, are both preferentially fed on by A. gambiae in comparison with fructose or glucose presented alone. In addition, sucrose is preferred over the monosaccharide mixture in feeding assays. By contrast, A. gambiae shows a lower appetence for glucose or fructose alone, monosaccharides which elicit weak responses from the sugarsensitive neurones in T1 sensilla. Nonetheless, we cannot affirm from our results whether the labellar taste neurones are able to detect glucose or fructose at concentrations fed on by $A$. gambiae in nature (i.e. higher than $100 \mathrm{mM}$ ). Furthermore, whereas this study focuses on the labella of $A$. gambiae, the presence of sugar-sensitive neurones capable of responding to glucose or fructose remains to be verified in gustatory sensilla on tarsi, at the labrum tip, on the inner surfaces of the labella and within the cibarium where taste neurones may occur (McIver 1982). Feeding choices made by insects do not just depend on the ability of a ligand to activate or inhibit peripheral taste neurones (Hassett et al. 1950). Insects also have post-ingestive pathways for assessing nutrient quality that can modulate feeding preferences (Burke and Waddell 2011; Fujita and Tanimura 2011; Wright 2011; Dus et al. 2011; Stafford et al. 2012). For example, despite sorbitol being tasteless for Drosophila, flies prefer to feed on $100 \mathrm{mM}$ sorbitol than on water. This response could be mediated by the internal expression of Grs, as Drosophila mutants lacking the DmGr43a in their brains do not feed on sorbitol over water (Miyamoto et al. 2012). Recently, the AgGr25 receptor of A. gambiae, an ortholog of DmGr43a in Drosophila, has been characterized (Freeman et al. 2014). Neurones expressing DmGr43a or AgGr25 respond strongly to fructose and to other sugars such as glucose and sucrose (Miyamoto et al. 2012; Freeman et al. 2014). Since both fructose and glucose are weak labellar sugar-sensitive neurone activators, the internal AgGr25 sugar receptor may have played a role in A. gambiae feeding on these two monosaccharides over water in the choice assays described here. This might suggest that Grs are necessary for post-ingestive feedback regarding nutrient quality in A. gambiae.

Although melezitose activates labellar sugar-sensitive neurones of A. gambiae, no significant reduction in the preference for sucrose was observed when the mosquitoes had the choice between sucrose and melezitose. In the winter mosquito $C$. inornata, sucrose is the best sugar feeding stimulant followed by melezitose and an equimolar mixture of glucose and fructose (Schmidt and Friend 1991). Glucose and fructose also show synergism in the feeding responses of the yellow fever mosquito, A. aegypti (Ignell et al. 2010), and of the blowfly Phormia regina (Dethier et al. 1956).

Sugar feeding preferences and their nutritive values for $A$. gambiae

Our findings show that A. gambiae with access to sucrose, the preferred sugar in our study, accumulate higher energetic reserves, as represented by the higher dry mass of females, and have a longer lifespan than those fed with glucose. In addition, the dry mass of females with access to glucose decreased between 4 and 10 days old, while that of females with access to sucrose increased in the same period. This increase in dry mass may reflect lipogenesis during the first 7 days in A. gambiae with ad libitum access to $10 \%$ sucrose (wt:vol) (Kaufmann and Briegel 2004).

We found that 4-day-old females previously fed on glucose will feed on glucose after one night of starvation but 10-day-old females hardly feed on glucose. Such a change in sugar-meal acceptance is not found in females fed on sucrose. It could be that the weak palatability of glucose to A. gambiae leads to a lower sugar intake by the mosquito, thus accelerating the drop in their energy reserves. No significant difference in lifespan was observed when $A$. gambiae females were fed sucrose or fructose as already observed in another mosquito species, Aedes communis (Andersson 1992). However, A. gambiae with access to the mixture of glucose and fructose, which is a stronger phagostimulant than fructose, survive significantly shorter than on fructose alone. Since glucose constitutes half of the molecules in the mixture, the difference in longevity of $A$. gambiae fed fructose over glucose could be due to a difference in nutritive value between the two monosaccharides. By contrast, in other Diptera, an equimolar mixture of fructose and glucose is as suitable for survival in Drosophila as sucrose (Hassett 1948) and in the winter mosquito $C$. inornata, sucrose, fructose and glucose support comparable values of longevity (Nayar and Sauerman 1975).

Sugar diet influences the biting behaviour of female $A$. gambiae

Female A. gambiae fed on glucose show a more intense biting behaviour on the heated glass disk than those fed on sucrose. In mosquitoes, free carbohydrates, including glucose, constitute the first fuel to sustain flight, followed by glycogen and lipids used for longer flights (Nayar and Sauerman 1971; Nayar and Van Handel 1971; Kaufmann and Briegel 2004). In the present study, the higher biting response recorded for females fed on glucose could be explained by the fact that glucose can be used immediately to sustain flight even though it constitutes a weaker substrate to support survival. Consequently, females fed 
on glucose would attempt to compensate for their lower energy state by actively seeking a blood meal, resulting in the more intensive biting behaviour recorded on the heated glass disk.

In conclusion, our study reveals that the sugar diet used to maintain laboratory mosquito colonies influences their energy reserves and thus impacts on longevity and on female biting behaviour. It is unlikely that mosquitoes will have access to nectar containing only glucose in a natural setting. We would predict, however, that the biting rate of A. gambiae would be higher when not supported by the correct proportion of glucose and fructose in its diet. Indeed, previous studies have shown that mosquitoes are more likely to forage for blood meals in mesocosms with plants producing nectar with low sugar concentrations (Stone et al. 2012). The present study suggests that mosquito longevity and biting behaviour depend not only on the abundance of sugar sources, but also on sugar quality. Nonetheless, nectar constituents are not confined to fructose, glucose or sucrose as tested here. Nectars are made up of complex mixtures of sugars (Manda et al. 2007b), amino acids (Vrzal et al. 2010) and secondary plant metabolites. These compounds, individually or combined, can differentially affect mosquito longevity and blood feeding behaviour, two main factors contributing to their vectorial capacity (Garrett-Jones 1964; Dye 1986).

Acknowledgments Radu Slobodeanu, Institute of Mathematics, University of Neuchâtel, is cordially acknowledged for his help with statistics. Our gratitude is also extended to Thomas Kröber, Animal Physiology Laboratory, University of Neuchâtel, for improvements to the biting assay in collaboration with Virgile Rumo, HE-ARC, Neuchâtel and Daniel Varidel, Technical Service, University of Neuchâtel. All members of the Animal Physiology Laboratory are acknowledged, particularly Martine Bourquin, for her help in mosquito rearing and Charles Chappuis, Alexandre Gurba as well as two anonymous reviewers for their worthwhile suggestions for improvements to the manuscript. The authors wish to thank Geraldine Wright, Institute of Neuroscience, Newcastle University, and Andrew McMullin, Institute of Biology, University of Neuchâtel for help with a revision of the manuscript. The Guinea pigs used for the mosquito rearing were maintained in an animal house under permit, in compliance with Swiss Federal Regulations. This research was funded by the Swiss National Science Foundation [Grant no. 138207].

\section{References}

Altner H, Prillinger L (1980) Ultrastructure of invertebrate chemo-, thermo-, and hygroreceptors and its functional significance. Int Rev Cytol 67:69-139

Andersson IH (1992) The effect of sugar meals and body size on fecundity and longevity of female Aedes communis (Diptera, Culicidae). Physiol Entomol 17:203-207

Burke CJ, Waddell S (2011) Remembering nutrient quality of sugar in Drosophila. Curr Biol 21:746-750

Crawley MJ (2007) The R book, 1st edn. John Wiley \& Sons Ltd, England
Dahanukar A, Lei YT, Kwon JY, Carlson JR (2007) Two Gr genes underlie sugar reception in Drosophila. Neuron 56:503-516

Dethier VG, Evans DR, Rhoades MV (1956) Some factors controlling the ingestion of carbohydrates by the blowfly. Biol Bull 111:204-222

Dus M, Min S, Keene AC, Lee GY, Suh GS (2011) Taste-independent detection of the caloric content of sugar in Drosophila. Proc Natl Acad Sci USA 108:11644-11649

Dye C (1986) Vectorial capacity: must we measure all its components? Parasitol. Today 2:203-209

Foster WA (1995) Mosquito sugar feeding and reproductive energetics. Annu Rev Entomol 40:443-474

Freeman GE, Wisotsky Z, Dahanukar A (2014) Detection of sweet tastants by a conserved group of insect gustatory receptors. Proc Natl Acad Sci USA 111:1598-1603

Fujita M, Tanimura T (2011) Drosophila evaluates and learns the nutritional value of sugars. Curr Biol 21:751-755

Garrett-Jones C (1964) Prognosis for interruption of malaria transmission through assessment of mosquitos vectorial capacity. Nature 204:1173-1175

Gary RE, Foster WA (2001) Effects of available sugar on the reproductive fitness and vectorial capacity of the malaria vector Anopheles gambiae (Diptera: Culicidae). J Med Entomol 38:22-28

Gary RE, Foster WA (2004) Anopheles gambiae feeding and survival on honeydew and extra-floral nectar of peridomestic plants. Med Vet Entomol 18:102-107

Hassett CC (1948) The utilization of sugars and other substances by Drosophila. Biol Bull 95:114-123

Hassett CC, Dethier VG, Gans J (1950) A comparison of nutritive values and taste thresholds of carbohydrates for the blowfly. Biol Bull 99:446-453

Heimpel GE, Jervis MA (2005) Does nectar improve biological control by parasitoids. In: Wäckers FL, van Rijn PC, Bruin J (eds) Plant-provided food for carnivorous insects: a protective mutualism and its applications, 1st edn. Cambridge University Press, New York, pp 267-304

Ignell R, Okawa S, Englund JE, Hill SR (2010) Assessment of diet choice by the yellow fever mosquito Aedes aegypti. Physiol Entomol 35:274-286

Kaufmann C, Briegel H (2004) Flight performance of the malaria vectors Anopheles gambiae and Anopheles atroparvus. J Vector Ecol 29:140-153

Kent LB, Robertson HM (2009) Evolution of the sugar receptors in insects. BMC Evol Biol 9:41

Kessler S, Vlimant M, Guerin PM (2013) The sugar meal of the African malaria mosquito Anopheles gambiae and how deterrent compounds interfere with it: a behavioural and neurophysiological study. J Exp Biol 216:1292-1306

Kröber T, Kessler S, Frei J, Bourquin M, Guerin PM (2010) An in vitro assay for testing mosquito repellents employing a warm body and carbon dioxide as a behavioral activator. J Am Mosq Control Assoc 26:381-386

Manda H, Gouagna LC, Nyandat E, Kabir EW, Jackson RR, Foster WA, Githure JI, Beier JC, Hassanali A (2007a) Discriminative feeding behaviour of Anopheles gambiae s.s. on endemic plants in western Kenya. Med Vet Entomol 21:103-111

Manda H, Gouagna LC, Foster WA, Jackson RR, Beier JC, Githure JI, Hassanali A (2007b) Effect of discriminative plant-sugar feeding on the survival and fecundity of Anopheles gambiae. Malar J 6:113

McIver SB (1982) Sensilla of mosquitoes (Diptera: Culicidae). J Med Entomol 19:489-535

Miyamoto T, Slone J, Song X, Amrein H (2012) A fructose receptor functions as a nutrient sensor in the Drosophila brain. Cell 151:1113-1125

MR4: Malaria Research and Reference Reagent Resource Center (2011) Method in Anopheles research, 2nd edn. pp 267-304. 
http://www.mr4.org/Portals/3/MR4_Publications/Anopheles\%20 Protocol\%20Manual\%20Second\%20Ed\%20v2011/2011\%20 Complete\%20Manual\%20PDF\%20TOC.pdf

Nayar JK, Sauerman DM (1971) Physiological effects of carbohydrates on survival, metabolism, and flight potential of female Aedes taeniorhynchus. J Insect Physiol 17:2221-2233

Nayar JK, Sauerman DM (1975) Effects of nutrition on survival and fecundity in Florida mosquitoes. Part 1. Utilization of sugar for survival. J Med Entomol 12:92-98

Nayar JK, Van Handel E (1971) The fuel to sustained mosquito flight. J Insect Physiol 17:471-481

Nyasembe VO, Teal PEA, Mukabana WR, Tumlinson JH, Torto B (2012) Behavioural response of the malaria vector Anopheles gambiae to host plant volatiles and synthetic blends. Parasit Vectors 5:234

Omand E, Dethier VG (1969) An electrophysiological analysis of the action of carbohydrates on the sugar receptor of the blowfly. Proc Nati Acad Sci USA 62:136-143

Pappas LG, Larsen JR (1976) Gustatory hairs on the mosquito, Culiseta inornata. J Exp Zool 196:351-360

Pappas LG, Larsen JR (1978) Gustatory mechanisms and sugarfeeding in the mosquito, Culiseta inornata. Physiol Entomol 3:115-119

Pinheiro J, Bates D, DebRoy S, Sarkar D, R Core Team (2014) Linear and nonlinear mixed effects models. R package version 3.1-117. http://CRAN.R-project.org/package $=$ nlme

R Development Core Team (2014) R: a language and environment for statistical computing. R Foundation for Statistical Computing, Vienna, Austria. ISBN 3-900051-07-0. http://www.r-project.org/
Rasband WS (2011) ImageJ, U.S. National Institutes of Health, Bethesda, Maryland, USA. http://imagej.nih.gov/ij/

Schmidt JM, Friend WG (1991) Ingestion and diet destination in the mosquito Culiseta inornata - effects of carbohydrate configuration. J Insect Physiol 37:817-828

Skaug H, Fournier D, Nielsen A, Magnusson A, Bolker B (2014) Generalized linear mixed models using AD model builder. $\mathrm{R}$ package version 0.8.0. http://glmmadmb.r-forge.r-project.org/ glmmADMB.html

Stafford JW, Lynd KM, Jung AY, Gordon MD (2012) Integration of taste and calorie sensing in Drosophila. J Neurosci 32:14767-14774

Stone CM, Hamilton IM, Foster WA (2011) A survival and reproduction trade-off is resolved in accordance with resource availability by virgin female mosquitoes. Anim Behav 81:765-774

Stone CM, Jackson BT, Foster WA (2012) Effects of plant-community composition on the vectorial capacity and fitness of the malaria mosquito Anopheles gambiae. Am J Trop Med Hyg 87:727-736

Therneau T (2014) Survival. R package version 2.37-7. http://cran.rproject.org/web/packages/survival/

Vrzal EM, Allan SA, Hahn DA (2010) Amino acids in nectar enhance longevity of female Culex quinquefasciatus mosquitoes. J Insect Physiol 56:1659-1664

Whitehead AT, Larsen JR (1976) Electrophysiological responses of galeal contact chemoreceptors of Apis mellifera to selected sugars and electrolytes. J Insect Physiol 22:1609-1616

Wright GA (2011) Appetitive learning: memories need calories. Curr Biol 21:R301-R302 